\title{
WHEN WOLVES RECOLONIZE: INDIRECT EFFECTS ON THE SMall ANd Medium-Sized Mammal Community
}

\author{
BRIAN MILLER $\uparrow$ DENVER ZOOLOGICAL FOUNDATION \\ DENVER $\uparrow \mathrm{CO}$ \\ HANK HARLOW $\uparrow$ DEPARTMENT OF ZOOLOGY \& PHYSIOLOGY \\ UNIVERSITY OF WYOMING $\uparrow$ LARAMIE
}

\begin{abstract}
$\uparrow \quad$ ABSTRACT
The abundance and diversity of mammals will be greatly affected by a number of factors, including plant productivity, climate, natural disturbance, and disease. Of particular interest to conservation strategies, there is little known about the ecological role that carnivores play in maintaining ecosystem structure. Large carnivores were essentially eliminated from much of their range during the last century. Yet, a growing body of experimental evidence indicates that top carnivores are keystone species, and they play important roles in maintaining the health of Nature. The predatory activities of large carnivores produce effects that ripple through the trophic levels of an ecosystem and affect organisms that seem distantly removed, ecologically and taxonomically. But, few studies have examined the indirect impacts of predation across those trophic levels. Such studies have been deemed a high priority in the Greater Yellowstone Ecosystem. Presently, we are assessing the abundance of selected species of mammals at sites representing five major vegetation types found in the Grand Teton National Park. The five vegetation sites are sampled in areas with and without wolves. The size range of these mammals extends from voles/mice to coyotes. Small rodents are being assessed through standard capture/recapture techniques using Sherman traps. Carnivores are being estimated by genetic identification of scat.
\end{abstract}

That method is non-invasive, and by walking transects two times, we can essentially estimate populations sizes using the Lincoln-Peterson statistical technique. Mammals that can not be easily trapped or identified through scat will be followed over time using indices of abundance.

\section{Problem Statement}

Despite Leopold's half-century old advice on "intelligent tinkering" we have not kept "every $\operatorname{cog}$ and wheel." Today, the scythe of extinction cuts 1000 times faster than historical background rates, and its pace is increasing. That scythe has dire implications. When "cogs or wheels" are lost, a system can fluctuate outside of the bounds to which it has adapted. Depending on which parts are lost, and the rate of loss, the pressure on a given system can exceed its ability to respond. We would like to address a specific category of such an event - the loss of carnivores and how the loss of their top-down forces has simplified ecosystems. Through our study, we are documenting the influence of recolonizing wolves on the structure and abundance of the small and medium-sized mammal community.

As an example, the recent recolonization of wolves (Canis lupus) in the northern Midwest has restricted the distance that beavers (Castor Canadensis) forage from aquatic habitats, and that reduces the impact of beaver on plant associations. 
The presence of wolves on Isle Royale, Michigan has also produced compelling evidence of a trophic cascade. Similarly, the re-establishment of wolves in other areas has been followed by declines in caribou (Rangifer tarandus), moose (Alces alces), elk (Cervus elephus), and deer (Odocoileus hemionus). These herbivores are themselves predators of plants. Because there are known linkages among herbivores, microbes, and soil nutrients, the impact of predation affects abundance, diversity, and biomass of plants and animals, and thus ecological interactions such as predation and competition.

It is crucial to try to understand the role of large carnivores in ecosystem health. Ecological research that analyzes such interactions is "imperative" in the Greater Yellowstone Ecosystem. If top predators play a role in maintaining the integrity of ecological communities, then managing them successfully will be critical. On the other hand, failure to understand their role, and manage accordingly, can result in distorted ecological interactions that will jeopardize biodiversity.

\section{$\downarrow \quad$ RESEARCH OBJECTIVES}

We are assessing the abundance and densities of selected species of mammals at sites representing five defined vegetation types found in the Grand Teton National Park. We now have three years of background data on these five habitat types in areas without wolves. In 2002, we will continue collecting data on those sites, but we will also collect data from the same five habitat types in areas with wolves. This approach will provide information on small and medium-sized mammals that will illuminate the impact of wolf colonization on the mammal community. Data will also provide the National Park Service with a background of information that will allow them to monitor changes in species composition as a result of any biotic and abiotic changes that may occur in the future.

\section{MeTHODS}

We censused small and medium-sized mammal populations in five vegetation types that followed the maps and definitions created by Debinski et al. Sites were recorded by UTMs and selected to be are located at the same altitude. We sampled 2 replicates in each habitat type both in the presence of wolves and in their absence. The habitats sampled included: (1) sagebrush, (2) mixed forb- grasses, (3) grass, sedge, and willow meadow, (4) wet meadow of grass and sedge, and (5) old-growth lodgepole pine.

We followed the standard capture-recapture techniques for small mammals using folding Sherman traps that are $22.5 \mathrm{~cm}$ long and 7.5 by 7.5 $\mathrm{cm}$ wide. Traps were opened late in the day and closed after checking the next morning. Each trap holds cotton bedding for the comfort of the animal. In three years of trapping, our trap-mortality has stayed consistently around $4 \%$.

Capture-recapture techniques involve capture, marking, recapture, and recording the proportion of marked to unmarked animals in the recapture. Average capture probability, or proportion of population represented in captures, can be estimated from capture records of marked animals.

We marked rodents with small alloy ear tags purchased from National Band and Tag. Marking with the small ear-tags has been used on other rodents, and we also used it on the endangered blackfooted ferret (Mustela nigripes). It is safe for the animal, reliable for the researcher, and the tag is inconspicuous (if a visitor should happen to see a mouse or vole in the field, which is highly unlikely). The tag would pose no threat to any carnivores eating a marked animal, as carnivores routinely eat bone fragments much larger than the tag. Ingested tags are passed through the carnivore gut and excreted in scat, or in the case of raptors, they are expelled in a pellet (pers. Obs.).

We trapped a site continuously until recaptures roughly equal new captures. The sampled area can be considered a closed population because the intervals between trapping periods are so short. Abundance is calculated with several estimators, including Lincoln-Peterson which extrapolates a simple ratio of unmarked captures per 1000 trapnights. Number of trap-nights is adjusted for sprung traps.

The population size associated with a grid is a function of two known factors (grid area and perimeter) and two unknown factors (boundary stripwidth and true animal density). So, data for each one-hectare grid will also be analyzed as a series of nested grids to address the issue of boundary stripwidth and make the population estimate more accurate. Analyses of nested grids also allows us to identify the smallest possible grid size that accurately reflects population estimate, via the law of diminishing returns (e.g. plotting an accumulation 
curve with population estimates from each grid size in the nested system).

Species presence/absence and an index thought to be correlated with abundance is taken on the 1 hectare plot. Northern pocket gopher (Thomomys talpoides) was estimated by counting mounds within the grid and badgers (Taxidae taxus) were estimated by number of fresh digs (presence of fresh sub-soil on the mound that is not yet hardened by sun).

We are estimating numbers of small and medium-sized carnivores by collecting feces, determining the genotype following the two techniques used by Kohn et al. to estimate coyote population numbers. Genetic analysis involves molecular typing with hypervariable microsatellite markers. Genetic work is being done at Aurora College in Denver, where Dr. Anna Goebel has a working laboratory and is presently extracting microsatellite markers from scat. Estimating population numbers is a very non-intrusive method of collecting reliable date. Dr. Goebel is presently applying the technique with 75 coyote and 120 pine marten scats that we collected walking approximately $200 \mathrm{k}$ ilometers of trails and stream beds in the 100 $\mathrm{km}^{2}$ study area in Grand Teton National Park. Microsatellites are published for coyotes and pine marten.

Tracking the impact of wolf colonization on small and medium-sized mammals over time in the Grand Teton National Park may be demonstrated with a time-series analysis, as was done when measuring the effects of sea otter (Enhydra lutris) reintroduction on selected species in that system. Time series analysis, and/or appropriate multivariate techniques, will allow examination of changes that occur as wolves arrive to the habitats we are sampling in the Grand Teton National Park. Simultaneous sampling of small and medium-sized mammals in areas with and without wolves will allow a spatial control to compliment temporal analysis.

We record temperature and precipitation in all areas sampled to judge their effect on mammal populations. With this information, we will compare composition of the mammal community in areas with and without wolves using multivariate statistical techniques. Correlations may also be used. Such analyses does not lend cause and effect, but they do lend important insight for inductive and retroductive reasoning, upon which hypotheses can be constructed and tested experimentally.

\section{Relevance to Conservation}

Land managers often are responsible for the maintenance of species diversity and resilience. This requires knowledge of ecosystem dynamics over decades and centuries. Resource-driven (bottom-up) models guided early actions on managing species and ecosystems. Under this paradigm, carnivores have little ecological value, and throughout the $20^{\text {th }}$ Century carnivore management strategies (often extirpation) have reflected that concept. An alternative hypothesis, however, states that herbivores reduce the biomass of plants, but in turn, the biomass of herbivores is checked by the presence of carnivores. As such, carnivores have great ecological value. Their predation activities create impacts that ripple downward through the trophic levels of an ecosystem. Here we examine how carnivores contribute to the structure and diversity of the small and medium-sized mammal community. The subtleties of these interactions have strong implications for management strategies of carnivores. Without considering these indirect impacts, shortsighted management strategies to reduce carnivores might cause extensive and long-term changes in ecosystem structure and function.

\section{Description of Deliverables}

1. A continuous, long-term data set on small and medium-sized mammals for the Grand Teton National Park. Before our work, such a data set did not exist in Grand Teton National Park.

2. A scientific publication on how recolonizing wolves can indirectly affect the small and medium-sized mammal community. This would lead to a better understanding of the role that carnivores play in ecosystem health.

3. A Master's thesis for a conservation biologist, Ms. Pam Elbers, who is studying at the University of Denver.

4. Data on interactions among small and medium-sized mammals and large carnivores should supply information useful in the design of both macro and micro-connections during regional reserve design. 